\title{
EchoGéo
}

15 | 2011

La Belgique, État prospère en décomposition au cœur de l'Europe du nord-ouest

\section{Les configurations de l'espace économique et la crise politique en Belgique}

\section{Christian Vandermotten}

\section{OpenEdition}

Journals

Édition électronique

URL : https://journals.openedition.org/echogeo/12359

DOI : 10.4000/echogeo.12359

ISSN : 1963-1197

Éditeur

Pôle de recherche pour l'organisation et la diffusion de l'information géographique (CNRS UMR 8586)

Référence électronique

Christian Vandermotten, «Les configurations de l'espace économique et la crise politique en Belgique », EchoGéo [En ligne], 15 | 2011, mis en ligne le 12 avril 2011, consulté le 03 août 2021. URL : http://journals.openedition.org/echogeo/12359; DOI : https://doi.org/10.4000/echogeo.12359

Ce document a été généré automatiquement le 3 août 2021.

EchoGéo est mis à disposition selon les termes de la licence Creative Commons Attribution - Pas d'Utilisation Commerciale - Pas de Modification 4.0 International (CC BY-NC-ND) 


\title{
Les configurations de l'espace économique et la crise politique en Belgique
}

\author{
Christian Vandermotten
}

\section{Une Belgique prospère en crise politique}

Avec un PIB/hab. supérieur en 2009 de $16 \%$ à la moyenne européenne, comme l'Allemagne, plus que la France ( $8 \%$ ), la Belgique fait figure de pays prospère au coeur de l'espace central européen. Les performances en termes de croissance sur les dix dernières années (2,2 \% par an entre 1999 et 2008) sont en phase avec celles de l'Union européenne $(2,3 \%)$ et de l'OCDE (2,5\%) (OCDE, 2010). La productivité du travailleur belge est exceptionnelle, plus forte que celle du travailleur américain si on considère les différentiels de taux d'occupation et de durée moyenne du travail (tableau 1).

Tableau 1 - Différentiels de productivité entre les travailleurs belges, français, allemands et américains (2009)

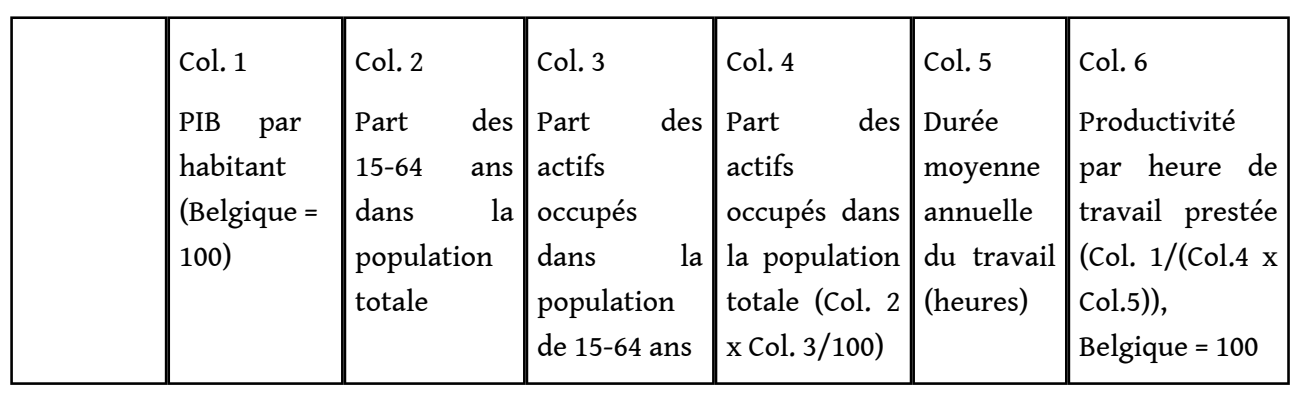




\begin{tabular}{|l|l|l|l|l|l|l|}
\hline Belgique & 100 & $66,0 \%$ & $61,6 \%$ & $40,7 \%$ & 1550 & 100 \\
France & 93 & $64,9 \%$ & $64,1 \%$ & $41,6 \%$ & 1554 & 91 \\
Allemagne & 100 & $65,8 \%$ & $70,9 \%$ & $46,7 \%$ & 1390 & 97 \\
Etats-Unis & 126 & $67,1 \%$ & $67,6 \%$ & $45,4 \%$ & 1768 & 99 \\
\hline
\end{tabular}

Sources: EUROSTAT; calculs de l'auteur.

2 L'insertion dans la CECA, le Marché commun, puis la Communauté et l'Union européenne ainsi que le glissement de l'économie belge vers une économie très tertiarisée ont encore accru son ouverture, historiquement très forte, dans un petit pays, au marché intérieur étroit, et dont l'industrie était spécialisée au XIXe siècle et dans la première moitié du XXe dans l'exportation de semi-finis. La somme des exportations de biens et de services représentait $27 \%$ du pib en 1953, $65 \%$ en 1974, $88 \%$ en 2007 , avec une part croissante due aux exportations de services ( $15 \%$ du total en $1953,18 \%$ en $1974,20 \%$ en 2007).

3 Même la crise récente a été relativement bien supportée par l'économie belge, quitte à placer plus encore son système financier sous contrôle étranger, avec la reprise de la principale banque belge, Fortis, par BNP Paribas. L'endettement public excessif (un maximum de 134,1 \% du PIB en 1993) est redescendu à 96,8 \% en 2010 (84,2 \% en 2007), et les créanciers sont largement nationaux.

4 Comment comprendre ce paradoxe d'une situation économique relativement confortable et d'une crise politique structurelle latente de l'État belge, qui s'est traduite par une succession de révisions constitutionnelles depuis 1970, débouchant aujourd'hui sur une crise aiguë, le pays fonctionnant depuis 300 jours (au 9 avril 2011) en régime d'affaires courantes, avec un gouvernement démissionnaire? 
Figure 1 - Répartition et spécificités de la production de la valeur ajoutée (2008)

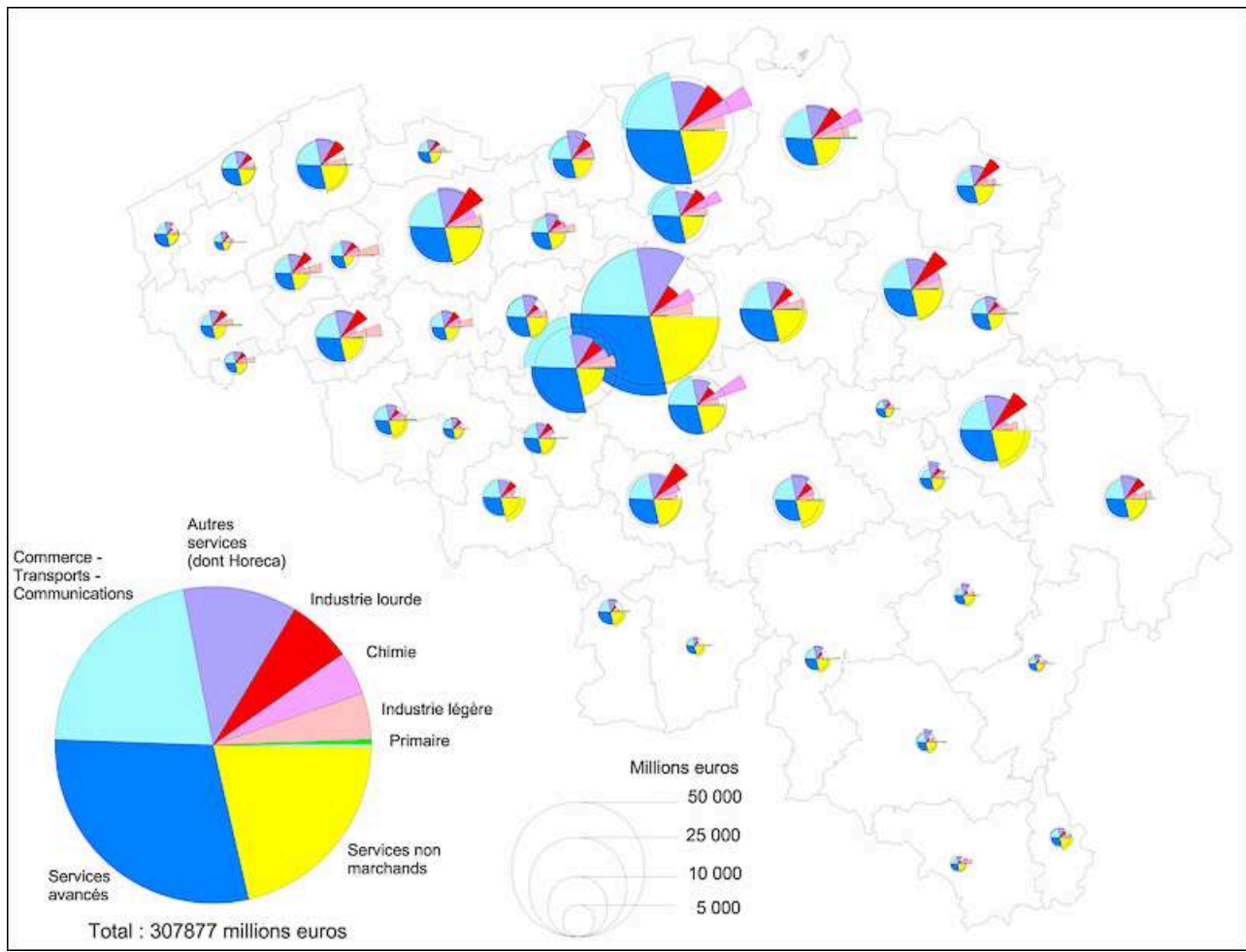

Les surfaces des cercles sont proportionnelles à la valeur ajoutée produite dans chaque arrondissement. L'angle au centre des secteurs est identique pour chaque cercle et correspond à la part de ce secteur dans la valeur ajoutée nationale. Le rayon des secteurs est donc ajusté de manière à ce que leurs surfaces soient proportionnelles à la valeur ajoutée de la catégorie d'activité. La longueur des rayons permet ainsi de juger des spécificités sectorielles de chaque arrondissement. Source : Banque Nationale de Belgique (BNB), réalisation IGEAT/ULB.

Ce délitement d'un État constitué tardivement (1830) s'inscrit dans le contexte d'une inversion historique de prospérité entre le Nord et le Sud du pays après la Seconde Guerre mondiale, dans le cadre des investissements massifs qui accompagnent la croissance rapide durant la phase $\mathrm{A}$ du $4{ }^{\text {ème }}$ cycle de Kondratieff : le Nord du pays, avec Bruxelles, a produit en $200976,5 \%$ du PIB, la Wallonie $23,5 \%$, pour respectivement $67,7 \%$ et $32,3 \%$ de la population (fig. 1). A l'inverse, en 1896, la Wallonie occupait $51,2 \%$ de l'emploi industriel et abritait $41,4 \%$ de la population. Mais au-delà, depuis plusieurs décennies, les clivages s'accentuent sur tous les plans entre la Flandre et le reste du pays: les comportements sociaux se différencient de plus en plus et la séparation linguistique de la presse et entre les corps électoraux fait qu'il n'y a plus d'opinion publique nationale. Même un sondage d'opinion révélant une très large volonté de maintien de l'existence de la Belgique recouvre des significations très différentes pour les francophones et les néerlandophones ${ }^{1}$.

Le processus de fédéralisation dissociative de l'État est entamé de fait dès les années soixante du siècle dernier, avec la fixation définitive de la «frontière linguistique » en 1962-1963. Ce processus a conduit à la transformation officielle de la Belgique en un État fédéral en 1993, moment qui apparaît avoir été une étape plutôt qu'un aboutissement. Dans la situation actuelle (comptes nationaux 2010), en tenant compte des charges d'intérêt et hors transferts budgétaires entre niveaux de pouvoir, l'État fédéral assure $23 \%$ des dépenses publiques, les Régions et Communautés $24 \%$, les 
pouvoirs locaux $13 \%$ et la sécurité sociale (fédérale) $40 \%$ (hors charges d'intérêts, ces pourcentages sont de $18 \%, 25 \%, 14 \%$ et $43 \%)$. Le débat actuel est dominé par les demandes flamandes de transferts massifs de compétences et de moyens financiers supplémentaires aux Régions et aux Communautés ${ }^{2}$ (par exemple en matière de politique de l'emploi et de sécurité sociale, ainsi que le renforcement d'une fiscalité propre de ces institutions), et en filigrane par les objectifs de disparition à terme de l'État belge au programme de la N-VA (Nieuw Vlaamse Alliantie, Nouvelle Alliance flamande), maintenant le premier parti en Flandre ${ }^{3}$ et au Parlement fédéral, sans parler de l'extrême-droite du Vlaams Belang ${ }^{4}$ (littéralement les Intérêts flamands).

\section{La posture scientifique}

7 Avec ce paradoxe, pays prospère, État en crise, en point de mire, l'objectif du présent article n'est ni de faire une géographie politique de la Belgique, ni d'en dresser la géographie économique. L'une et l'autre sont bien documentées ${ }^{5}$. Ce n'est pas non plus d'examiner l'ensemble des fractures sociales qui traversent l'espace belge, très souvent en se superposant au clivage linguistique, bien exposées dans un ouvrage qui fait référence (Groupe Mort-Subite, 1990).

Il s'agit plutôt d'éclairer le rôle déterminant joué en dernier ressort par l'économie dans les attitudes et les évolutions politiques, dès le XIX ${ }^{\mathrm{e}}$ siècle. Mais l'expression en dernier ressort est ici essentielle, faute de tomber dans un déterminisme réducteur et caricatural.

Ce cheminement politique peut en effet être articulé aux spatialités de l'économie du pays, dans les temps longs et dans leurs configurations actuelles, tant dans la sphère des réalités objectives que dans celle de leur représentation idéologique et du ressenti. Mais la compréhension de cette articulation impose de bien prendre en compte que l'importance des médiatisations idéologiques et politiques est importante au point que les causes économiques premières peuvent ne plus être perçues comme telles, et ceci d'autant plus que ces médiatisations politiques, identitaires communautaires, possèdent une temporalité propre et parfois bien plus longue que celle des réalités économiques qui les ont générées.

\section{La crise séculaire de la Flandre, aux origines des caractéristiques idéologiques dominantes du mouvement flamand}

On peut donc ainsi comprendre le paradoxe d'une Flandre contemporaine, qui défend des positions politiques qui sont celles d'une région prospère peu soucieuse de partage de ses ressources avec la Wallonie, mais sur la base de positions idéologiques fondées dans les temps longs d'une frustration culturelle d'une région pauvre, rurale, économiquement et politiquement périphérique, alors surpeuplée, du moins dans les zones textiles - provinces de Flandre orientale et occidentale. Cette pauvreté s'était fortement aggravée, au moment même où la Wallonie entamait une brillante révolution industrielle, du fait de la crise de l'économie textile flamande, au milieu du XIX ${ }^{e}$ siècle, avec l'effondrement du textile à domicile, dans un contexte libre-échangiste ${ }^{6}$. Le grand capitalisme belge s'intéressait peu au textile, laissant plutôt la gestion difficile du 
textile flamand, peu concurrentiel, à un capitalisme local émietté de petits et moyens entrepreneurs, dans un cadre dominant de paternalisme semi-rural (sauf à Gand). Cette situation de crise structurelle latente, avec le maintien de l'importance du textile dans les structures économiques, a perduré jusqu'après la Première, voire la Seconde Guerre mondiale.

11 Cette crise, laissant pour compte les campagnes et les zones semi-rurales de la Flandre, a donné naissance aux caractères dominants (mais non exclusifs) du flamingantisme, en tant que mouvement conservateur, anti-urbain, interclassiste ${ }^{7}$, communautariste, luttant pour la pleine reconnaissance linguistique du néerlandais et de l'identité culturelle (c. Kesteloot \& F. De Maesschalk, 2001). L'histoire du mouvement flamand est donc fondée sur un sentiment d'infériorité culturelle, une volonté de reconquête pas à pas d'une reconnaissance identitaire et linguistique, se nourrissant à des racines mythologiques médiévales ${ }^{8}$, puis, à partir de l'entre-deux-guerres, une affirmation progressive d'une nouvelle bourgeoisie flamande, aux dépens de l'ancienne bourgeoisie francophone, "fransquillonne », des grandes villes de Flandre (M. Quévit, 1978, 2010). Ces sentiments persistent alors même que les Flamands dominent aujourd'hui les rouages de l'État fédéral, en raison de leur nombre et de leur prospérité économique.

\section{La crise économique wallonne et les évolutions spatiales et structurelles de l'économie belge durant la phase fordiste}

A la sortie de la Seconde Guerre mondiale, l'économie belge, peu détruite par la guerre et très rapidement opérationnelle, avec le port d'Anvers tombé intact aux mains des Alliés, le seul dans ce cas entre Le Havre et Hambourg, conservait encore pour l'essentiel ses structures sectorielles héritées du xix siècle et leur géographie (tableau 2). C'était une économie dominée par les grands holdings nationaux, au premier rang desquels la Société Générale, une économie industrielle, spécialisée dans les semi-finis le marché intérieur était limité, d'autant que les salaires belges étaient bas jusqu'à la Première, voire jusqu'à la Seconde Guerre mondiale. Les fleurons moteurs en étaient donc toujours en Wallonie, même si la dynamique industrielle flamande était déjà supérieure depuis la fin du XIX ${ }^{e}$ siècle, surtout dans l'aire métropolitaine centrale, entre Bruxelles et Anvers, dont les activités portuaires ont donné lieu à l'implantation d'importantes activités industrielles en aval dès les années 1880 .

Tableaux 2 - Évolution de la ventilation régionale de la valeur ajoutée (1955-2009)

Tableau 2A - Part dans l'ensemble de l'économie

\begin{tabular}{|l|l|l|l|l|l|}
\hline & 1955 & 1970 & 1990 & $2000(f)$ & $2009(f)$ \\
\hline Région flamande & $\mathbf{4 8 , 5}$ & $\mathbf{5 3 , 8}$ & $\mathbf{5 9 , 0}$ & $\mathbf{5 7 , 3}$ & $\mathbf{5 7 , \mathbf { 1 }}$ \\
\hline dont Flandre occidentale et orientale (b) & 20,9 & 22,6 & 24,3 & 21,8 & 21,8 \\
\hline dont Anvers et Limbourg (b) & 20,9 & 23,6 & 27,2 & 25,3 & 24,9 \\
\hline
\end{tabular}




\begin{tabular}{|l|l|l|l|l|l|}
\hline Région wallonne & $\mathbf{3 3 , 5}$ & $\mathbf{2 9 , 1}$ & $\mathbf{2 5 , \mathbf { 1 }}$ & $\mathbf{2 3 , 5}$ & $\mathbf{2 3 , 5}$ \\
\hline dont Hainaut et Liège (c) & 26,0 & 22,1 & 17,9 & 15,7 & 15,2 \\
\hline \hline dont Brabant wallon & 1,8 & 2,0 & 2,3 & 3,1 & 3,6 \\
\hline Bruxelles-Capitale & $\mathbf{1 8 , 0}$ & $\mathbf{1 7 , 1}$ & $\mathbf{1 5 , 9}$ & $\mathbf{1 9 , 0}$ & $\mathbf{1 9 , 4}$ \\
\hline Aire métropolitaine bruxelloise (d) & 26,5 & 26,7 & 25,7 & 32,4 & 33,3 \\
\hline \hline Royaume & $\mathbf{1 0 0 , 0}$ & $\mathbf{1 0 0 , 0}$ & $\mathbf{1 0 0 , 0}$ & $\mathbf{1 0 0 , 0}$ & $\mathbf{1 0 0 , 0}$ \\
\hline
\end{tabular}

Tableau 2B - Part dans l'industrie extractive et manufacturière (a)

\begin{tabular}{|l|l|l|l|l|l|}
\hline & 1955 & 1970 & 1990 & $2000(f)$ & $2009(f)$ \\
\hline Région flamande & $\mathbf{4 6 , 3}$ & 57,7 & $\mathbf{6 8 , 0}$ & $\mathbf{7 0 , 5}$ & $\mathbf{6 8 , 4}$ \\
\hline dont Flandre occidentale et orientale (b) & 18,3 & 23,6 & 27,0 & 26,7 & 26,6 \\
\hline dont Anvers et Limbourg (b) & 22,1 & 26,5 & 35,2 & 36,2 & 34,0 \\
\hline Région wallonne & $\mathbf{4 0 , 1}$ & $\mathbf{3 2 , 5}$ & $\mathbf{2 2 , 0}$ & $\mathbf{2 2 , 3}$ & $\mathbf{2 5 , 0}$ \\
\hline dont Hainaut et Liège (c) & 34,1 & 26,5 & 17,2 & 15,8 & 16,2 \\
\hline dont Brabant wallon & 2,2 & 2,3 & 1,9 & 3,2 & 5,5 \\
\hline Bruxelles-Capitale & $\mathbf{1 3 , 4}$ & $\mathbf{9 , 8}$ & $\mathbf{1 0 , 0}$ & $\mathbf{7 , 2}$ & $\mathbf{6 , 6}$ \\
\hline Aire métropolitaine bruxelloise (d) & 21,5 & 19,7 & 17,7 & 17,9 & 20,0 \\
\hline Royaume & $\mathbf{1 0 0 , 0}$ & $\mathbf{1 0 0 , 0}$ & $\mathbf{1 0 0 , 0}$ & $\mathbf{1 0 0 , 0}$ & $\mathbf{1 0 0 , 0}$ \\
\hline
\end{tabular}

Tableau 2C - Part dans les services financiers (e)

\begin{tabular}{|l|l|l|l|l|l|}
\hline & 1955 & 1970 & 1990 & $2000(f)$ & $2009(f)$ \\
\hline Région flamande & $\mathbf{2 4 , 7}$ & $\mathbf{3 3 , 1}$ & $\mathbf{4 0 , 2}$ & $\mathbf{3 4 , 6}$ & $\mathbf{3 0 , 4}$ \\
\hline dont Flandre occidentale et orientale (b) & 6,6 & 12,2 & 14,4 & 10,9 & 9,5 \\
\hline dont Anvers et Limbourg (b) & 12,9 & 16,4 & 18,9 & 17,0 & 15,0 \\
\hline Région wallonne & $\mathbf{1 2 , 5}$ & $\mathbf{1 4 , 4}$ & $\mathbf{1 4 , 4}$ & $\mathbf{1 2 , 4}$ & $\mathbf{1 2 , 2}$ \\
\hline dont Hainaut et Liège (c) & 10,8 & 11,4 & 11,0 & 8,8 & 8,2 \\
\hline
\end{tabular}




\begin{tabular}{|l|l|l|l|l|l|}
\hline dont Brabant wallon & 02 & 05 & 06 & 1,4 & 1,6 \\
\hline Bruxelles-Capitale & $\mathbf{6 2 , 7}$ & $\mathbf{5 2 , 5}$ & $\mathbf{4 5 , 5}$ & $\mathbf{5 3 , 0}$ & 57,3 \\
\hline Aire métropolitaine bruxelloise (d) & 68,1 & 57,4 & 53,0 & 61,1 & 64,9 \\
\hline \hline Royaume & $\mathbf{1 0 0 , 0}$ & $\mathbf{1 0 0 , 0}$ & $\mathbf{1 0 0 , 0}$ & $\mathbf{1 0 0 , 0}$ & $\mathbf{1 0 0 , 0}$ \\
\hline
\end{tabular}

(a) Y compris les garages en 1955 et 1970.

(b) Très grossièrement, la Flandre orientale et occidentale correspondent plutôt à l'aire de développement des PME, Anvers et le Limbourg à celle de l'industrialisation portuaire et pétrochimique et fordiste de montage, cette dernière plus spécifique en Limbourg, en localisation plus périphérique.

(c) Ces deux provinces incluent l'ancien axe charbonnier et industriel lourd wallon.

(d) En première approximation, Bruxelles-Capitale, Brabant flamand et Brabant wallon ( = ancienne province de Brabant). Y compris donc dans le Brabant flamand, Louvain, dont le bassin d'emploi reste assez autonome par rapport à celui de Bruxelles.

(e) La localisation des services financiers est la plus spécifique de celle du commandement économique. La catégorie statistique des services aux entreprises couvre un champ trop vaste, allant de services réellement directionnels à d'autres qui sont plus ubiquistes (activités de nettoyage, de maintenance, etc.).

(f) Les séries ne sont pas parfaitement comparables avec celle de 1990 et antérieures, du fait d'une meilleure prise en compte des différentiels régionaux de salaires et de qualification de la main-d'œuvre dans la comptabilité régionale, ce qui revalorise les chiffres bruxellois et affaiblit ceux des deux autres Régions. Toutefois, si elle amplifie quelque peu les tendances observées entre 1990 et 2000, cette revalorisation ne remet pas en cause leur interprétation.

Sources: INS, Comptabilités régionales; y compris calculs et estimations de l'auteur pour des désagrégations sur la base des recensements industriels de 1947 et de 1961. Ces chiffres n'incluent pas, pour Bruxelles, l'activité des ambassades et des organisations internationales à statut extraterritorial (sauf pour leur personnel non statutaire qui dépendrait de la sécurité sociale belge).

Le redémarrage très rapide de l'économie à la sortie de la guerre et les besoins en charbon et en semi-finis des économies européennes en reconstruction n'ont pas incité le grand capital belge à procéder à des reconversions sectorielles massives. Dès lors, la crise charbonnière qui intervient à partir du milieu des années cinquante, en conséquence de la faible rentabilité des gisements et de la réorientation de la base énergétique européenne vers les hydrocarbures, va frapper de plein fouet une Wallonie qui n'y était pas préparée. Les gisements charbonniers y étaient moins rentables qu'en Campine, où les veines étaient plus épaisses et moins faillées et qui, du fait d'une mise en valeur plus tardive, disposaient d'un équipement plus moderne. La crise charbonnière dans les bassins wallons va en outre être suivie de difficultés dans des secteurs métallurgiques lourds, comme les constructions ferroviaires, en attendant plus tard la crise de la sidérurgie. La chimie wallonne, axée sur la carbochimie, a aussi été atteinte de plein fouet.

14 Tout cela se déroule dans un contexte de désengagement industriel du grand capital national. A l'inverse, les capitaux étrangers affluent massivement en Belgique à partir de 1959, aidés par les premières lois dites d'expansion économique. Mais ils investissent en majorité dans le Nord du pays, soit en localisation portuaire, à Anvers, avec un développement remarquable de la pétrochimie, soit dans les bassins de main-d'œuvre qui subsistent dans le Nord-est, où la démographie était restée plus longtemps vigoureuse. L'industrie de type fordiste, en particulier la construction automobile, y profite d'une localisation intermédiaire entre le port d'Anvers et la Ruhr, bien desservie par le canal Albert et par l'ouverture précoce de l'autoroute Roi Baudouin. Simultanément, l'industrie de petites et moyennes entreprises, très présente dans 
l'Ouest de la Flandre et y formant des districts industriels, comme autour de Courtrai, profite des aides accordées à la reconversion des zones textiles.

On assiste donc à la mise en place d'une alliance entre une nouvelle bourgeoisie industrielle flamande, les structures d'encadrement du capitalisme international des multinationales et le personnel politique flamand, de plus en plus dominant dans les rouages de l'administration nationale, qui favorise le développement du Nord du pays, dans un contexte politique dominé par l'interclassisme du parti social-chrétien flamand. On comprend donc qu'à l'inverse, le ressentiment de la classe ouvrière wallonne envers le grand capitalisme industriel belge traditionnel, dont les sièges sont très majoritairement implantés à Bruxelles depuis le XIX siècle et qui a désinvesti l'ancien sillon industriel wallon pour se tourner de plus en plus vers les placements financiers, se soit exacerbé, au moment même où l'essor économique s'accélérait dans le Nord du pays (tableau 3).

Tableau 3 - Différentiels entre le taux de croissance économique régional annuel et celui du Royaume

\begin{tabular}{|l|l|l|l|}
\hline & Flandre & Wallonie & Bruxelles-Capitale \\
\hline $1955-1959$ & $+0,4 \%(\mathrm{a})$ & $-1,0 \%$ & $+0,7 \%(\mathrm{a})$ \\
$1959-1964$ & $+0,8 \%(\mathrm{a})$ & $-1,3 \%$ & $+0,5 \%(\mathrm{a})$ \\
$1966-1970$ & $+1,4 \%$ & $-1,2 \%$ & $-2,5 \%$ \\
$1970-1974$ & $+0,8 \%$ & $-0,6 \%$ & $-1,8 \%$ \\
$1975-1984$ & $+0,6 \%$ & $-0,5 \%$ & $-1,5 \%$ \\
$1985-1993$ & $+0,3 \%$ & $-0,3 \%$ & $-0,4 \%$ \\
$1994-1997$ & $+0,5 \%$ & $-0,5 \%$ & $-0,9 \%$ \\
$1999-2009$ & $+0,0 \%$ & $-0,0 \%$ & $+0,2 \%$ \\
\hline
\end{tabular}

(a) L'arrondissement périphérique de Hal-Vilvorde est comptabilisé avec Bruxelles.

Sources: INS et BNB; calculs de l'auteur.

Les difficultés économiques qui frappent la Wallonie à partir de la crise charbonnière dans la seconde moitié des années cinquante génèrent des revendications politiques fédéralistes qui s'expriment largement sur des fondements de classe explicites, dans une région où la classe ouvrière était fortement organisée par les piliers du mouvement socialiste, plutôt que sur une base identitaire, comme en Flandre'. Ceci a entretenu côté flamand le mythe du Wallon gréviculteur et assisté, allant aujourd'hui jusqu'à la revendication égoïste au démantèlement des mécanismes fédéraux de transfert sociaux. Ainsi, à l'inverse de la Flandre, la temporalité de l'émergence politique de la revendication fédéraliste est côté wallon simultanée à celle des problèmes économiques et directement et explicitement reliée à ceux-ci.

Par ailleurs, la base de classe de la revendication wallonne implique aussi initialement une absence de solidarité politique entre la Wallonie et Bruxelles, perçue comme la « ville des capitalistes » malgré la similitude linguistique et l'exacerbation des querelles à ce propos, qui aboutit dès 1963 à donner un caractère définitif à la limite linguistique, enserrant donc la capitale dans un anneau de communes flamandes, dont six bénéficient toutefois d'un régime dit «de facilités». 


\section{De 1974 au début des années 1990, une consolidation de la position dominante de la Flandre}

Malgré le ralentissement de la croissance industrielle et l'accélération de la tertiarisation de l'économie à partir de 1974 (tableau 4), l'avantage comparatif de la Flandre va se maintenir sur sa lancée (tableau 3). D'une part, la base industrielle y est plus récente et donc moins sujette à rationalisations; d'autre part le tissu de petites et moyennes entreprises, bien développé en Flandre, résiste mieux à la crise. Le niveau de tertiarisation des grandes villes flamandes, qui bénéficient de leur pesanteur historique, est qualitativement supérieur à celui des villes wallonnes, pour beaucoup des conurbations industrielles formées au XIX ${ }^{\mathrm{e}}$ siècle, à l'exception notable de Liège, où l'économie urbaine est plus basée, par défaut, suite à la désindustrialisation, sur le secteur non-marchand. Le port d'Anvers et l'industrialisation portuaire, avec le raffinage ${ }^{10}$ et la pétrochimie, soutiennent l'activité dans le Nord du pays. Enfin, la croissance plus rapide des revenus flamands, dont le niveau par habitant dépasse ceux des wallons à partir de 1970 (et ceux des habitants de la Région de Bruxelles-Capitale à partir de 1988) assure un développement plus vigoureux du commerce et des services aux personnes.

Tableau 4 - Quelques indicateurs significatifs de l'évolution de la structure sectorielle de l'économie belge

\begin{tabular}{|l|l|l|l|l|l|}
\hline & 1953 & 1970 & 1990 & 2002 & 2007 \\
\hline Agriculture, sylviculture, pêche & 7,6 & 4,1 & 2,2 & 1,2 & 0,8 \\
\hline Industrie extractive (a) & 4,3 & 3,4 & 0,2 & 0,1 & 0,1 \\
\hline Textile et confection & 4,5 & 3,3 & 1,5 & 1,0 & 0,7 \\
\hline Chimie & 2,5 & 3,8 & 4,7 & 4,9 & 4,5 \\
\hline Métallurgie, machines et fabr. de véhicules & 10,4 & 13,2 & 9,6 & 6,7 & 6,3 \\
\hline Autres industries (b) & 12,5 & 8,9 & 6,6 & 6,0 & 4,9 \\
\hline Total industrie manufacturière & 29,8 & 29,3 & 22,5 & 18,6 & 16,4 \\
\hline Transports, logistique, communications & 6,4 & 6,7 & 7,0 & 7,0 & 8,2 \\
\hline Services aux entreprises, financiers et assurances & 12,5 & 15,0 & 22,8 & 27,9 & 29,1 \\
\hline Services non marchands et aux personnes & 17,5 & 18,1 & 22,6 & 24,6 & 23,3 \\
\hline Autres (c) & 21,9 & 23,4 & 22,7 & 20,6 & 22,2 \\
\hline
\end{tabular}


(a) Essentiellement l'extraction charbonnière. Le dernier puits wallon est fermé en 1984 ; en 1992, c'est le tour du dernier puits campinois - zone où l'extraction n'avait débuté qu'en 1917, sous une épaisse couverture de morts-terrains

(b) Industries agro-alimentaires, bois et meuble, minéraux non métalliques, etc.

(c) Construction, eau, gaz, électricité, commerce, hôtels-restaurants.

L'industrie extractive s'effondre avant la fin de la période fordiste (milieu des années soixante-dix), de même que la part la plus importante du textile, de la confection et des autres industries manufacturières. Le déclin relatif de la métallurgie se poursuit jusque dans les années quatre-vingtdix ; il ne concerne pas seulement la métallurgie de base, mais aussi de larges pans des fabrications métalliques et mécaniques. Même l'industrie automobile et des implantations fordistes des années soixante sont fragilisées. Seule la chimie progresse relativement de manière tendancielle, avec une mutation interne, de la carbochimie vers la pétrochimie, puis vers la chimie fine, en particulier les industries pharmaceutiques. Jusqu'au début des années quatre-vingt, la croissance relative des services marchands était surtout liée à celle du secteur financier ; par la suite elle l'est à l'explosion des services aux entreprises.

Sources : d'après les chiffres de l'INS et de la BNB.

La reconversion de l'axe wallon est difficile, tant du fait des pesanteurs sociologiques dans une zone de vieille industrialisation que des héritages, des inerties, d'un environnement dégradé, $d$ 'une faiblesse des villes wallonnes en matière de services de haut niveau qualitatif, dans une région qui ne maîtrisait pas le commandement de son économie, malgré son ancienne prospérité. Et cela d'autant plus que la croissance économique globale est modeste. Il en est résulté une situation difficile sur le plan social et sur celui du marché du travail côté wallon.

\section{Bruxelles, de la grande ville industrielle à la petite ville mondiale}

20 Jusque dans les années soixante, outre les fonctions tertiaires liées à son statut de capitale et de centre financier et de commandement économique national, Bruxelles était la plus grande des villes industrielles belges.

21 A partir du milieu des années soixante, malgré son insertion internationale croissante, la position relative de Bruxelles s'est toutefois dégradée, tout en restant forte. Les développements du tertiaire dans la capitale, avec entre autres une forte croissance du secteur financier, étaient alors compensés par la désindustrialisation massive, qui fait passer le nombre de personnes occupées dans l'industrie manufacturière de 163000 en 1970 à 60000 en 1990 (il en reste 24000 en 2008). Encore, parmi cet emploi dit industriel qui subsiste, $44 \%$ étaient formés en 1990 d'employés, pour beaucoup dans des sièges sociaux sans activité de production ( $52 \%$ en 2008). Certes, cette stagnation bruxelloise, d'ailleurs partagée à l'époque par la plupart des autres grandes villes européennes, était partiellement compensée par une forte croissance de la périphérie, en particulier flamande, qui accueillait certaines délocalisations de l'industrie bruxelloise. Mais la croissance périphérique était surtout le fait de nouveaux développements logistiques et tertiaires. Globalement, même en additionnant à Bruxelles les deux arrondissements périphériques de Hal-Vilvorde (flamand) et du Brabant wallon, l'emploi industriel a régressé entre 1970 et 1990 de 247000 à 113000. 

mondial - et elle l'est d'autant moins que le grand capital belge s'est désengagé de l'industrie comme on l'a vu, et que la finance belge elle-même s'est fondue dans la finance française et néerlandaise, seule la flamande Kredietbank restant une importante banque belge dont le siège est à Bruxelles. Parmi les 2000 principales firmes mondiales, tous secteurs d'activité confondus (classement Forbes 2006), seules 12 sont belges. Leurs sièges sont tous implantés dans l'aire métropolitaine centrale belge, neuf à Bruxelles, une dans la banlieue bruxelloise flamande (à Hal), une à Louvain et deux à Anvers. Soit $65 \%$ du chiffre d'affaire de ces firmes contrôlés au départ de Bruxelles, $69 \%$ si on y ajoute Hal. Ces grandes firmes belges sont sousreprésentées dans le classement mondial et européen, si on se réfère au développement du pays et par comparaison à des pays de petite taille mais qui ont conservé un commandement capitaliste national: le contrôle sur les 2000 plus grandes firmes mondiales exercé au départ de la Belgique ne concerne que 0,54\% de leur chiffre d'affaire total (tableau 5).

Tableau 5 - La faiblesse de la Belgique dans le commandement du grand capital européen

\begin{tabular}{|l|l|l|}
\hline & $\begin{array}{l}\text { Part dans le PIB européen (UE à 27) } \\
\text { (à prix courants, aux taux de } \\
\text { change) }\end{array}$ & $\begin{array}{l}\text { Part dans le chiffre d'affaire des 2 000 principales } \\
\text { firmes mondiales dont le siège est dans un pays de } \\
\text { l'UE à 27 }\end{array}$ \\
\hline \hline Belgique & $2,72 \%$ & $0,54 \%$ \\
\hline $\begin{array}{l}\text { Pays-Bas } \\
\text { (a) }\end{array}$ & $4,35 \%$ & $12,10 \%$ \\
\hline Suède & $3,39 \%$ & $3,53 \%$ \\
\hline Finlande & $1,45 \%$ & $2,01 \%$ \\
\hline
\end{tabular}

\section{(a) Y compris deux firmes anglo-néerlandaises. Sources : EUROSTAT et FORBES (2006).}

Mais en revanche, Bruxelles est devenue un nœud majeur des réseaux des firmes de services avancés, pour lesquelles il est essentiel d'avoir une forte présence dans la capitale européenne ${ }^{11}$. Les institutions européennes et internationales génèrent de manière directe, induite ou à travers les effets multiplicateurs de l'ordre de 90000 des 700000 emplois sur le territoire de Bruxelles-Capitale. Le tourisme d'affaire et de congrès ${ }^{12}$ a encouragé le développement d'un très important potentiel hôtelier ; il est de plus en plus complété par un tourisme de loisirs de courts séjours. Certes, beaucoup reste à faire pour mieux assurer la promotion internationale de Bruxelles - longtemps et parfois encore handicapée par la complexité des institutions bruxelloises -, mais il n'en reste pas moins que Bruxelles est devenue une ville majeure dans les classements internationaux, malgré sa taille relativement limitée ${ }^{13}$ (1 100000 habitants, maintenant en forte croissance, pour la Région elle-même; de l'ordre de 2800000 habitants pour 
l'ensemble du bassin d'emploi) (figure 2). La dynamique bruxelloise diffuse sur l'ensemble de l'économie belge et l'articule, en particulier le losange flamand central dont les sommets sont Anvers, Louvain, Bruxelles et Gand, ainsi que sur le Brabant wallon (figure 3). En même temps qu'à une tertiarisation accélérée, on assiste donc à une remétropolisation de l'économie belge, au profit de l'aire centrale. Cette remétropolisation n'englobe néanmoins pas les deux grandes villes wallonnes, Liège et Charleroi, mal insérées dans les réseaux de services, bien que celles-ci bénéficient toutefois de l'essor récent de deux aéroports régionaux, Liège-Bierset, spécialisé dans le trafic de fret, devenu huitième aéroport européen de ce point de vue, et CharleroiBrussels South, spécialisé dans le trafic low-cost, qui s'est hissé en 2010 à un trafic de 5,1 millions de passagers (pour 17 à 18 millions à Bruxelles-National (Zaventem), situé en Région flamande, immédiatement au Nord-est de Bruxelles).

Figure 2 - Aire de recrutement des migrants alternants vers le centre d'emploi bruxellois et évolution de la navette de travail entre 1991 et 2001

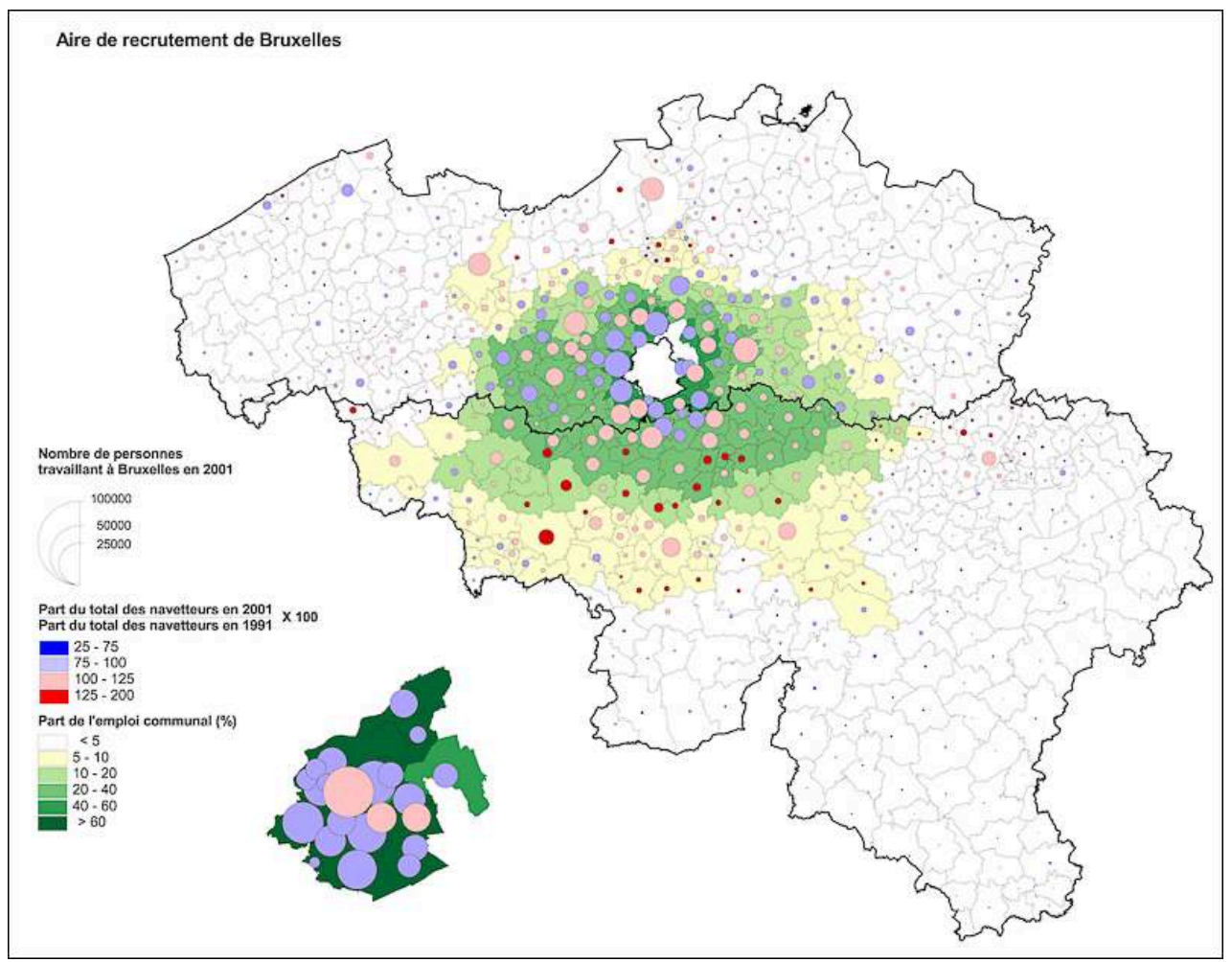

Source : INS, Enquête socio-économique de 2001. 
Figure 3 - Les villes belges dans le réseau national de services avancés aux entreprises

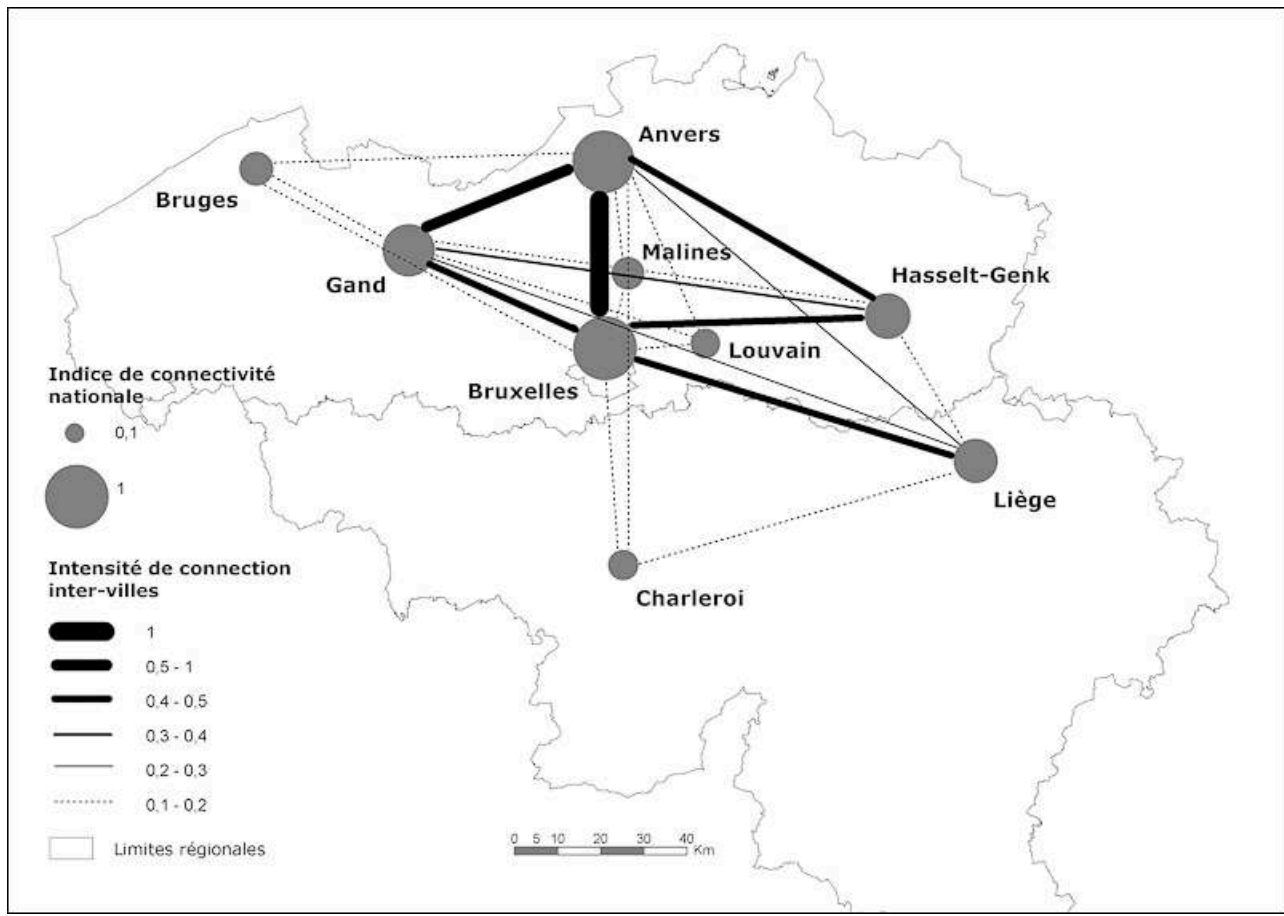

La carte suggère l'intensité des connectivités fonctionnelles inter-villes sur la base des localisations des sièges et des filiales des firmes de services avancés aux entreprises, selon une méthodologie inspirée de celle du GaWC (в. Derudder \& p.J. Taylor, 2003). Si, au niveau des connectivités intranationales, Bruxelles et Anvers sont proches, il n'en va pas de même au niveau des connectivités internationales : le niveau de connectivité d'Anvers tombe alors à $38 \%$ de celui de Bruxelles et Gand, Hasselt et Liège sont très nettement en-dessous du niveau de $20 \%$. On remarquera sur la carte la forte opposition entre le maillage serré du Nord du pays et la grande faiblesse des interconnections entre les deux principales villes wallonnes, preuve à nouveau de l'insuffisance des services marchands dans une Région dont l'industrie fut historiquement contrôlée au départ de Bruxelles et dont les deux bassins de Liège et du Hainaut partageaient les mêmes spécialisations extractives et lourdes, et connaissent les mêmes types de problématique de reconversion.

Source : Aujean L. \& al, 2007

Ceci éclaire l'ambiguïé des attitudes flamandes envers Bruxelles, à la fois proclamée capitale de la Flandre et ville mal aimée, francophone et cosmopolite, mais qui est, en tant que capitale européenne ${ }^{14}$ et seule ville véritablement internationale du pays, avec des services avancés de haut niveau qualitatif, le moteur de l'économie belge postmoderne, et donc aussi de larges pans de l'économie flamande. Bruxelles-Capitale, sur son minuscule territoire de $161 \mathrm{~km}^{2}$, produit $19,4 \%$ du PIB belge (plus de $20 \%$ si on tient compte des activités d'organismes internationaux non comptabilisées). Près de $60 \%$ de ses postes de travail sont occupés par des migrants alternants qui proviennent à raison d'un tiers environ de Wallonie, principalement du Brabant wallon, et des deux tiers de Flandre, en particulier du Brabant flamand et de l'Est de la Flandre orientale (figure 2). De la sorte, les revenus disponibles pour les habitants de Bruxelles ne représentent que $8,3 \%$ du revenu fiscal imposable belge. La contribution de la capitale à la dynamique économique flamande est donc considérable, à travers un transfert massif de revenus, d'une part, en tant que source du dynamisme de la périphérie, voire de pans entiers de l'économie nationale, de l'autre. Ceci fait de Bruxelles un enjeu majeur.

L'internationalisation de l'économie bruxelloise génère dans la capitale d'importants problèmes sociaux: elle $\mathrm{y}$ renforce d'autant plus les fractures sociales que les 
populations actives de classes moyennes et aisées ont largement migré vers la périphérie - donc en dehors des limites régionales (ou y habitaient déjà, en particulier pour celles originaires de Flandre - et que les populations pauvres d'origine immigrée résidant à Bruxelles ${ }^{15}$ n'ont pas les niveaux de formation, ni ne maîtrisent le multilinguisme, qui leur permettraient de postuler aux emplois de haut niveau qualitatif créés par l'économie internationalisée (figure 4). La prospérité économique bruxelloise retrouvée créé d'ailleurs relativement peu d'emplois, moins que ne le suggère la croissance de la valeur ajoutée : ces emplois sont à haute productivité, dans le secteur des services, les croissances quantitatives étant plus fortes en périphérie où se développent les activités logistiques, la maintenance, les back-offices, des laboratoires de recherche, etc. (tableau 6). De la sorte, les taux de chômage à BruxellesCapitale sont très élevés, surtout parmi les jeunes (19,5\% des actifs en situation de demande d'emploi en 2009 , pour 7,9\% dans la périphérie et $11,3 \%$ en moyenne belge). Bruxelles-Capitale offre donc ce paradoxe peu enviable d'être une ville à l'économie prospère, mais largement peuplée de populations pauvres ou fragilisées. Une situation d'autant plus difficile que les ressources de la Région dépendent pour une large part de la base fiscale de sa population résidente, alors que leur usage bénéficie largement aux navetteurs qui fréquentent chaque jour la capitale. Le refinancement de Bruxelles est donc aussi un enjeu majeur des négociations politiques : la Région de Bruxelles-Capitale revendique des ressources accrues structurelles, qui ne dépendent pas du bon vouloir du pouvoir fédéral, et donc de la majorité flamande. 
Figure 4 - Part des résidents bruxellois parmi les actifs occupés dans la Région de BruxellesCapitale, selon le niveau de diplôme

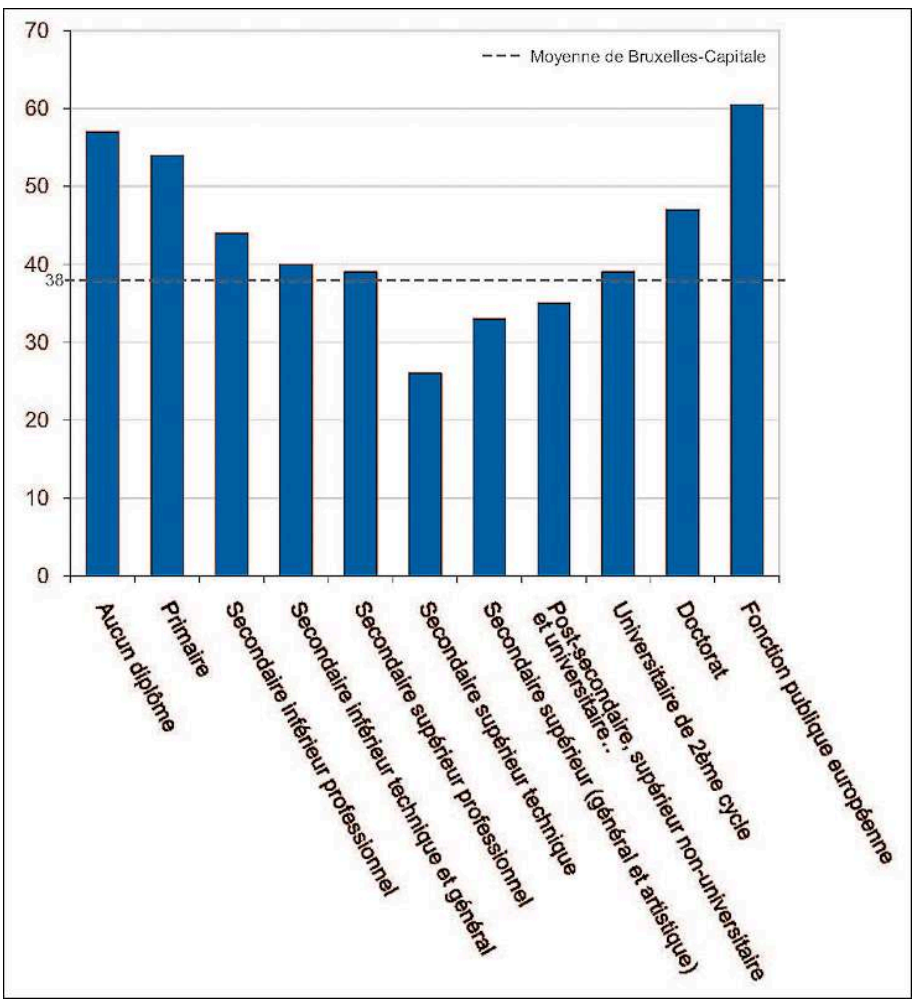

Les données pour les fonctionnaires européens sont présentées indépendamment de leur niveau de diplôme. Le graphique illustre bien la dualisation sociale bruxelloise, les classes moyennes étant les plus amenées à se périurbaniser pour disposer d'un logement de qualité à des prix acceptables pour elles.

Source : INS, Enquête socio-économique de 2001

Tableau 6 - Elasticité de la croissance de l'emploi à la croissance économique (2000-2009, calcul sur les bornes de la période)

\begin{tabular}{|l|l|l|}
\hline & $\begin{array}{l}\text { Taux de croissance annuel } \\
\text { moyen du PIB }\end{array}$ & $\begin{array}{l}\text { Elasticité de la croissance de l'emploi } \\
\text { à celle du PIB (a) }\end{array}$ \\
\hline Bruxelles-Capitale & $+1,6 \%$ & 0,36 \\
\hline $\begin{array}{l}\text { Arrondissements } \\
\text { périphériques (b) }\end{array}$ & $+2,1 \%$ & 0,60 \\
\hline Région métropolitaine & $+1,8 \%$ & 0,47 \\
\hline Flandre & $+1,3 \%$ & 0,73 \\
\hline $\begin{array}{l}\text { dont } \\
\text { (arrondissement) }\end{array}$ & $+0,7 \%$ & 1,26 \\
\hline dont Gand (arrondissement) & $+1,5 \%$ & 0,84 \\
\hline Wallonie & $+1,2 \%$ & 0,77 \\
\hline
\end{tabular}




\begin{tabular}{|l|l|l|}
\hline dont Liège (arrondissement) & $+0,9 \%$ & 0,74 \\
\hline $\begin{array}{l}\text { Dont Charleroi } \\
\text { (arrondissement) }\end{array}$ & $+0,6 \%$ & 1,33 \\
\hline Royaume & $+1,3 \%$ & 0,66 \\
\hline
\end{tabular}

(a) Rapport entre le taux de croissance annuel moyen de l'emploi et celui du PIB.

(b) Hal-Vilvorde et Brabant wallon.

Sources: Banque Nationale de Belgique, Comptabilité nationale.

\section{Quel avenir économique pour la Flandre et la Wallonie?}

27 A partir des années 1990, l'économie belge s'inscrit donc de plus en plus dans une mondialisation qui s'accompagne d'une accélération $\mathrm{du}$ processus de désindustrialisation. Celui-ci touche petit à petit l'économie des zones industrielles périphériques flamandes elles-mêmes, dont la dynamique ralentit. Dans celles dominées par les établissements contrôlés par des multinationales, des incertitudes se dessinent. Même l'industrie automobile est fragilisée, fermeture de Renault à Vilvorde en 1997, d'Opel à Anvers en 2010, réductions d'emploi et interrogations sur l'avenir à long terme de Ford à Genk. Les districts industriels de petites et moyennes entreprises de l'Ouest de la Région flamande semblent mieux résister, mais il n'est pas sûr qu'ils pourront continuer sur le long terme leur processus de remontée des filières technologiques sur la base des capitaux et des ressources technologiques et humaines disponibles au niveau d'entreprises locales, ni supporter les conséquences de la concurrence internationale dans des secteurs de l'industrie légère.

28 La chimie, pétrochimie et chimie fine, se porte bien, mais dans des localisations périmétropolitaines: zone portuaire anversoise, Campine anversoise, grande banlieue bruxelloise verte du Brabant wallon. Ces zones péri-métropolitaines sont aussi des lieux privilégiés de développement des activités logistiques et des secteurs technologiques de pointe.

29 A partir du début du XXI ${ }^{e}$ siècle, on peut observer des frémissements positifs dans la reconversion industrielle wallonne. Les efforts des pouvoirs publics commencent à porter certains fruits, d'autant que le foncier est plus aisément disponible et moins cher qu'en Flandre, beaucoup plus densément peuplée. Mais les nouvelles implantations sont surtout localisées au Nord de l'ancien axe industriel, le long de l'autoroute de Wallonie, de sorte que la redynamisation du vieux tissu urbain, dans lequel l'industrie traditionnelle était imbriquée, et l'assainissement des friches industrielles restent des problèmes majeurs. Surtout, l'amélioration relative des performances économiques wallonnes par rapport au Nord du pays ces dernières années, est largement liée à celles du Brabant wallon, dans la grande aire métropolitaine bruxelloise, induites par la périurbanisation et l'essor d'un pôle technologique à proximité de la capitale et de l'université de Louvain-la-Neuve, voire à une dynamique namuroise générée par le nouveau statut de cette ville comme capitale régionale ainsi qu'à l'essor de petites entreprises et au soutien à la consommation 
induits par l'extension du bassin d'emploi grand-ducal sur l'Est de la province du Luxembourg.

\section{Conclusions}

La Wallonie, dont la prospérité économique a largement assuré des transferts financiers en faveur de la Flandre à travers le $\mathrm{XIX}^{\mathrm{e}}$ et la première moitié $\mathrm{du} \mathrm{XX}^{\mathrm{e}}$ siècle, bénéficie certes aujourd'hui de la solidarité nationale. Mais en fait, les transferts actuels proviennent pour l'essentiel de la dynamique induite à partir de l'économie bruxelloise, alors qu'en termes de revenu par habitant de ses résidents la capitale est descendue en 2008 à l'indice 83,5, par rapport à une moyenne belge de 100, moins que la Wallonie $(94,2)$ (la Flandre est à l'indice 106,1). Par ailleurs, le différentiel de croissance entre la Flandre et la Wallonie s'est pratiquement réduit à zéro au cours des dernières années. La Flandre nationaliste développe donc aujourd'hui, comme en Italie du Nord, un discours de refus de solidarité avec le Sud du pays, lié à son statut de région prospère et dominante, mais qui s'inscrit sur des bases idéologiques qui trouvent leur fondement dans un passé ancien de faiblesse économique et d'oppression linguistique. Ceci à un moment où la mondialisation risque de remettre en question les avantages de dynamique acquis à partir des années soixante. En effet, l'industrie flamande risque de connaître des lendemains moins favorables : son appareil industriel mis en place durant les années soixante commence à son tour à vieillir; elle n'est plus à l'abri des impacts de la mondialisation sur ses industries fordistes, ni même sur son tissu de PME. A terme aussi, le vieillissement de la population devrait plus affecter la Flandre que la Wallonie, Bruxelles caracolant en tête en termes de jeunesse de sa population, du fait de l'immigration récente et de ses impacts sur la fertilité ${ }^{16}$. Contrairement à ce qu'avance la mythologie politique flamande, il n'est donc pas sûr qu'à moyen terme une disparition des mécanismes de transfert entre les Régions belges et de la solidarité inter-personnelle assurée par la sécurité sociale, dans une Belgique éclatée ou réduite à l'exercice d'un minimum de fonctions régaliennes, serait avantageux pour le Nord du pays. D'autant qu'il conviendrait de mesurer l'impact d'un tel éclatement sur la position internationale de Bruxelles et sur la dynamique globale qu'elle induit, y compris celle de la Flandre. D'où les sentiments schizophrènes de cette dernière envers Bruxelles, "sa » capitale mal aimée, à contenir impérativement dans ses étroites limites, mais en même temps dont elle ne veut (ne peut) se séparer.

31 A l'inverse, dans les dernières années, dans un contexte où de larges portions du monde politique wallon prennent de plus en plus conscience, cinquante ans après le début d'une dramatique désindustrialisation, de la nécessité d'appuyer aussi le redéploiement régional $\mathrm{du}$ Sud du pays sur les pôles tertiaires extra-régionaux (Bruxelles, mais aussi Luxembourg, voire Lille), et aussi en réponse à l'intransigeance flamande croissante, un renforcement de la solidarité entre la Wallonie et Bruxelles émerge: cela s'est concrétisé le 4 avril 2011 par le changement de nom de la Communauté française en Fédération Wallonie-Bruxelles, en dépit de l'appellation constitutionnelle officielle et au grand mécontentement de la Flandre, qui ne veut accepter le plein statut régional de Bruxelles, dont elle revendique la cogestion, et qui voit dans cette nouvelle dénomination une provocation politique.

Un État, même (très) amaigri, peut-il subsister quand sa capitale est à la fois une pomme de discorde et son (unique?) ciment? 


\section{BIBLIOGRAPHIE}

Aujean L., Castiau E., Roelandts M. \& Vandermotten C., 2007. Le positionnement des villes belges dans le réseau global des services avancés. Belgeo, 8, 1, p. 15-29.

Corijn E., Decroly J.M., Swyngedouw E. \& Vandermotten C., 2009. Note de synthèse $n^{\circ} 13$. Bruxelles, ville internationale. Etats généraux de Bruxelles. Brussels Studies, www.brusselsstudies.be

De Groof R. (ed.), 2008. Brussels and Europe. Bruxelles et l'Europ. Antwerpen, Academic and Scientific Publishers.

Delwit P., (2 éd., 2010. La vie politique en Belgique de 1830 à nos jours. Bruxelles, Éditions de l'Université de Bruxelles, coll. UBlire.

Derudder B. \& Taylor P.J., 2003. The global capacity of Belgium's major cities: Antwerp and Brussels compared. Belgeo, 4, 4, p. 459-476.

Groupe Mort-Subite (Kesteloot C., De Lannoy W., Saey P., Swyngedouw E. \& Vandermotten C.), 1990. Les fractionnements sociaux de l'espace belge. Une géographie de la société belge. Bruxelles, Contradictions, 58-59, p. 1-224.

IGEAT (ULB) (DIR.), 2007. Espon project 1.4.3, Study on urban functions, Final report, http:// www.espon.eu

Kesteloot C. \& De Maesschalk F., 2001. Anti-urbanism in Flanders: the political and social consequences of a spatial class struggle strategy. Belgeo, 2, 1-2, p. 41-62.

Marissal P., Medina Lockhart P., Van Hamme G., Kesteloot C. \& Vandermotten C., 2007. Monographie $n^{\circ}$ 6. Emploi et structures socio-économiques régionales. SPFEconomie, Politique scientifique fédérale.

Mérenne-Schoumaker B., Decroly J.M., Vandermotten C., Vanneste D. \& Verhetsel A. (dir.), 2011. Atlas de Belgique, tome 5, Géographie économique. Commission de l'Atlas du Comité national de Géographie, Politique scientifique fédérale, Gent, Academia Press.

Quévit M., 1978. Les causes du déclin wallon. Bruxelles, Vie ouvrière.

Quévit M., 2010. Flandre-Wallonie. Quelle solidarité ?. Charleroi, Couleurs livres.

Vandermotten C., 1985. La production de l'espace industriel belge : 1846-1984. Lille, Hommes et Terres du Nord, p. 100-109.

Vandermotten C., 2007. La crise de l'Etat belge. EchoGéo, Sur le vif 2007, http:// echogeo.revues.org/2049

Vandermotten C. \& Lennert M., 1999. Les structures spatiales de l'économie belge et leur évolution, de la période fordiste à aujourd'hui. Lille, Hommes et Terres du Nord, p. 186-197.

Vandermotten C. \& Roelandts M., 2006. Globalization and Social dualization, under an Institutional Constraint: The Brussels-Capital Case. Built Environment, 32, 2, p. 148-156.

Vandermotten C., Leclercq E., Cassiers T. \& Wayens B., 2009. Note de synthèse $n^{\circ} 7$. L'économie bruxelloise. Etats généraux de Bruxelles. Brussels Studies, www.brusselsstudies.be

Vandermotten C., Marissal P. \& Van Hamme G., $2^{\mathrm{e}}$ éd., 2010. La production des espaces économiques. Bruxelles, Éditions de l'Université de Bruxelles, 2 vol. 
Vandermotten C. \& Vandeburie J., $2^{\mathrm{e}}$ éd., 2011. Territorialités et politique. Bruxelles, Éditions de l'Université de Bruxelles.

Van der Wee H. \& Blomme J. (ed.), 1997. The economic development of Belgium since 1870. Cheltenham, Elgar Reference Collection.

Willaert D., 2010. De recente internationalisering van het Brussels gewest en de Vlaamse randgemeenten. Interface Demography, vub, Working paper 2010-2, http://www.vub.ac.be/demography

\section{NOTES}

1. Lors d'un sondage d'opinion mené durant la précédente crise politique, en $2007,88 \%$ des Wallons et $61 \%$ des Flamands s'exprimaient en faveur d'un maintien de la Belgique.

2. Rappelons que la Belgique fédérale est divisée en trois Régions (la Région flamande, BruxellesCapitale et la Région wallonne) et trois Communautés (flamande, française et germanophone), responsables exclusives respectivement pour les matières à inscription territoriale et pour celles relevant du statut et des services aux personnes, au premier rang desquelles l'enseignement et la culture. Il n'y a donc pas pour ces matières de compétences d'arbitrage dévolues au niveau fédéral, à la différence de ce que connaissent en général les systèmes fédéraux. Mais les choses se compliquent encore dans la mesure où la Communauté flamande a fusionné son Exécutif avec celui de la Région flamande. Ces deux institutions fusionnées ont en conséquence implanté leur capitale à Bruxelles, c'est-à-dire sur le territoire d'une autre Région. Elles se désignent avec ambiguïté sous le terme commun de "Vlaanderen» (Flandre). Du côté flamand, une part majoritaire de l'échiquier politique estime en fait que Bruxelles-Capitale n'est pas une Région à part entière (bien que ce statut lui ait été octroyé en 1989 par la Constitution) et qu'elle devrait être cogérée par les deux Communautés, française et flamande. Par ailleurs, le côté flamand considère que la Communauté flamande bénéficie de compétences exclusives sur le territoire de la Région flamande, y compris donc dans les communes dites "à facilités linguistiques ", en particulier six communes de la périphérie bruxelloise à nette majorité francophone. Les Flamands envisagent ces facilités comme un régime transitoire destiné à permettre l'adaptation des francophones, alors que les francophones les voient comme un droit définitivement acquis. La Flandre entend ainsi par exemple y contrôler le fonctionnement des écoles francophones et exige la réitération à chaque demande, et non pas une fois pour toutes, de l'obtention de documents administratifs en français.

3. Ce qui tend en outre à radicaliser les positions $\mathrm{du} C D \& V$ (les chrétiens-démocrates flamands) que la N-VA a supplanté dans sa position historiquement dominante sur l'échiquier politique du Nord du pays (31,7 \% des voix flamandes pour la N-VA au Sénat en 2010, contre 16,2 \% seulement pour les sociaux-chrétiens).

4. Suite aux élections législatives de 2010, les trois partis flamands ayant à leur programme la dissolution de l'Etat belge (la N-VA, le VB et la liste Dedecker, du nom d'un dissident libéral) recueillent $47,3 \%$ des voix dans le collège électoral flamand au Sénat, soit $29,2 \%$ des voix au niveau national (ce chiffre est monté à $49,2 \%$ dans un sondage mené fin mars 2011). Certes, cela ne signifie sans doute pas que la moitié des électeurs flamands seraient d'emblée favorables à une disparition de la Belgique, mais un enlisement de la crise pourrait faire rapidement évoluer les opinions de ce point de vue. En outre, les autres partis flamands réclament aussi, avec plus ou moins de virulence et d'intensité, des transferts importants de compétences du niveau fédéral vers celui des Régions et Communautés.

5. Pour la géographie politique, une synthèse exhaustive est fournie par le récent volume 1 de la troisième édition de l'Atlas national de Belgique (Marissal P. \& al., 2007) ou dans Vandermotten C. \& Vandeburie J. (2011). L'histoire politique du pays est développée dans Delwit P. (2010). La 
géographie économique l'est dans le volume 5 du même atlas (Mérenne-Schoumaker B. \& al., 2011), ainsi que dans Vandermotten C., Marissal P. \& Van Hamme G. (2010, vol. 2).

6. Le passé économique brillant de la Flandre médiévale avait déjà été préalablement cassé par la Contre-réforme, dans la seconde moitié du XVIe siècle, qui a vu une part importante de la bourgeoisie urbaine flamande passée au protestantisme s'exiler aux Pays-Bas. La hiérarchie des villes flamandes est toujours similaire en 1800 à ce qu'elle était au XVIe siècle et leur population est du même ordre de grandeur.

7. D'autant plus que l'émergence plus tardive de la classe ouvrière flamande, à un moment où le monde catholique s'était déjà doté de structures d'encadrement de celle-ci (suite à l'encyclique De Rerum Novarum, 1891), a permis le maintien majoritaire de son encadrement syndical et social par l'Eglise, à la différence de la Wallonie.

8. Et catholiques pour la tendance historique dominante du mouvement flamand, mais il y eut aussi, dès le milieu du XIX ${ }^{\mathrm{e}}$ siècle, un flamingantisme urbain libéral.

9. Le slogan des fédéralistes wallons durant les grèves de 1'hiver 1960-1961 est «Pour un fédéralisme démocratique et des réformes de structure anti-capitalistes ».

10. Les raffineries anversoises ne sont plus alimentées en pétrole par l'Escaut, dont le tirant d'eau ne permet pas le passage des plus gros pétroliers, mais par un pipe-line en provenance de Rotterdam.

11. La Générale de Banque et l'ancienne Caisse Générale d'Epargne et de Retraites, fusionnées en Fortis en 1993, ont été absorbées par BNP Paribas en 2009 ; le Crédit Communal a fusionné avec le Crédit Local de France en 1996, devenant Dexia ; la Banque Bruxelles-Lambert est passée aux mains de la néerlandaise ING en 1998.

12. Bruxelles est la première ville européenne et la deuxième mondiale pour l'organisation de congrès internationaux.

13. Dans le classement proposé par l'étude ESPON 1.4.3, Londres et Paris, les deux villes mondiales européennes, bénéficient d'un classement sur base de leur niveau de fonctionnalité de 9,7 et de 9,5; les quatre villes suivantes sont, dans un intervalle resserré, Rome, Madrid, Amsterdam et Bruxelles (entre 8,4 et 8,1 ). La même étude calcule un indice d'internationalisation : Paris et Londres sont toujours très nettement en tête (respectivement aux indices 107,5 et 100,0) ; viennent ensuite Francfort (40,7), Amsterdam (39,9), Madrid $(35,2)$, Munich $(32,2)$, Milan $(31,6)$, Zürich $(29,0)$ et Bruxelles $(26,4)$. Dans le classement du GaWC, fondé sur la connectivité dans les réseaux mondiaux de services avancés en 2000, Londres est à 1,000 (le maximum mondial), Paris à 0,699 , puis dans un étroit intervalle viennent Milan, Madrid, Amsterdam, Francfort et Bruxelles (entre 0,604 et 0,557$)$, devant Zürich $(0,485)$.

14. De l'ordre de 13 à $14 \%$ du PIB bruxellois réel - c'est-à-dire en y incluant les activités qui ne relèvent pas de la compatibilité nationale belge du fait de leur statut international - provient de manière directe ou induite de la présence dans la capitale des institutions européennes, du siège de l'OTAN et des nombreuses ambassades (E. Corijn \& al., 2009).

15. En 2006, la population de Bruxelles-Capitale est formée pour $28 \%$ de personnes de nationalité étrangère. Mais en fait, si on compte les populations ayant récemment acquis la nationalité belge ou dont les parents n'étaient pas belges, on arrive à $55 \%$ de personnes étrangères ou d'origine étrangère récente, sans doute un record européen. Parmi celles-ci, $22 \%$ environ sont originaires ou ressortissantes de pays « riches» (pour l'essentiel l'ancienne Union européenne), et $33 \%$ de pays « pauvres » (y compris le Centre-est et l'Est de l'Europe), les personnes d'origine marocaine, turque ou congolaise étant beaucoup plus massivement de nationalité belge (à raison de plus de $60 \%$ du total) que celles d'origine ouest-européenne (20 à $25 \%$ d'entre elles), nord-américaine ou japonaise (de l'ordre de $10 \%$ d'entre elles) (D. Willaert, 2010).

16. L'indice conjoncturel de fécondité était en 2007 de 1,77 enfants par femme en Région flamande, de 1,82 en Wallonie (mais de 2,09 à Bruxelles, du fait de l'importance de la population 
d'origine immigrée). D'après les Perspectives de population 2007-2060 du Bureau fédéral du Plan (2008), la part des 65 ans et plus dans la population, à peu près identique dans les trois Régions du pays en $2008(16,8 \%)$ passerait en 2030 à $22,1 \%$ en Wallonie et à $24,3 \%$ en Flandre (elle régresserait à $15,6 \%$ à Bruxelles).

\section{RÉSUMÉS}

L'inscription de la Belgique dans un processus continu de fédéralisation dissociative ne peut se comprendre qu'à travers une analyse des structures économiques du pays, à la fois sur les temps longs et dans le présent. L'idéologie identitaire qui domine en Flandre s'est développée en réaction à la crise séculaire de l'économie flamande entre le milieu du XIX ${ }^{e}$ siècle et l'entre-deuxguerres, ainsi qu'à l'arrogance francophone de la bourgeoisie nationale, mais aussi de celle des villes flamandes à l'époque. La crise de l'économie wallonne après la Seconde Guerre mondiale, similaire à celle de bien d'autres bassins d'ancienne industrialisation charbonnière et métallurgique, alors que la Région flamande connaissait un essor industriel, a généré dans le Nord du pays le sentiment que le Sud du pays devenait un fardeau pour son développement. Malgré le partage très majoritaire de l'usage du français, la Wallonie ouvrière a mis longtemps avant de prendre conscience de l'importance d'une solidarité économique (et politique) avec Bruxelles, perçue comme « la ville des capitalistes ». En revanche, la Flandre considère Bruxelles comme sa capitale mais a peu d'empathie pour une ville francophone et cosmopolite. Toutefois, dans le cadre de la globalisation, Bruxelles apparaît comme le moteur de l'économie belge tertiarisée, ce qui en fait à la fois une pomme de discorde dans la vie politique belge, mais peutêtre aussi un ciment pour le maintien d'une Belgique, dont les compétences qui resteront fédérales s'affaibliront en tout état de cause.

Belgium's political evolution is part of a process of dissociative federalization. This can only be understood with reference to the country's economic structures and their long-term trend. The community ideology prevailing in Flanders was developed as a reaction to the secular crisis of the Flemish economy, from the mid- $19^{\text {th }}$ Century to the inter-war, and to the arrogance of the French-speaking national and Flemish upper class of that time. The economic crisis that affected Wallonia and many European early coal-mining and metallurgical areas after World War II generated in Flanders the feeling that Wallonia had become a burden for its own development, characterized in turn by strong manufacturing growth. Even if a very large majority of Brussels' population is French-speaking (at least in the public sphere), working-class Wallonia took long to become aware of the importance of an economic and political solidarity with the capital, seen as "the city of capitalists". Inversely, the Flemish Region considers Brussels as its own capital despite it doesn't like its French-speaking and cosmopolitan character. However, in the framework of globalization, Brussels appears to be the driving force behind Belgium's tertiarized economy. As such, the capital is both a source of discord in the political life and a possible cement for Belgium. But even if Belgium survives, the federal powers will undoubtedly grow weaker. 
INDEX

Keywords : Belgium, Brussels, economic geography, economic situation, federalism, Flanders, Wallonia

Mots-clés : Belgique, Bruxelles, fédéralisme, Flandre, géographie économique, situation économique, Wallonie

\section{AUTEUR}

\section{CHRISTIAN VANDERMOTTEN}

Christian Vandermotten, Université libre de Bruxelles. cvdmotte@ulb.ac.be 